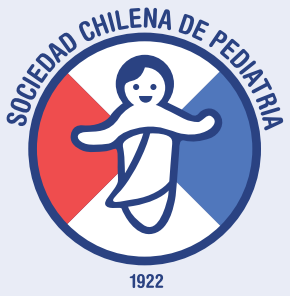

Rev Chil Pediatr. 2020;91(2):304-305

DOI: 10.32641/rchped.v91i2.2208
Revista Chilena
De PeDiatría

www.revistachilenadepediatria.cl

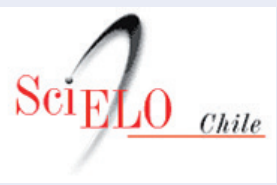

www.scielo.cl

\section{Prescribiendo ejercicio físico en períodos de cuarentena por COVID-19: ¿Es útil la autorregulación perceptual en niños?}

Dosing physical exercise intensity during the COVID-19 quarantine: Does perceptual self-regulation work in children?

\section{Sr. Editor}

La pandemia por coronavirus (COVID-19) aparecida inicialmente en China (Wuham) el pasado mes de diciembre 2019 se ha transformado en el principal problema de salud pública en el mundo, con 1.249.107 casos diagnosticados y una tasa de letalidad de $5,4 \%$ al momento de escribir esta carta (5 de abril de 2020) ${ }^{1}$.

En este contexto, los gobiernos de diversos países han debido tomar medidas protectoras para proteger a la población. En el caso de Chile, el gobierno ha implementado medidas progresivas y prudentes basadas en la dinámica epidemiológica de la pandemia, promoviendo el distanciamiento social mediante la reducción de viajes, solicitando a las personas permanecer en sus casas, cerrando escuelas y lugares de alta concentracion. Inevitablemente, todas estas medidas protectoras promueven el comportamiento sedentario de las personas, lo cual se asocia entre otros efectos, con un deterioro del estado fisico y el desarrollo de enfermedades cardiovasculares.

Es sabido que la condición física se reduce de forma precoz una vez que la vida sedentaria se establece, con consecuencias fisiopatológicas que incluyen la pérdida de masa muscular, obesidad, insulino-resistencia, disfunción endotelial, entre otros ${ }^{2}$. Por consiguiente, resulta perentorio promover acciones para prevenir las complicaciones secundarias a la inactividad física, mientras se respetan las medidas de cuarentena y aislamiento establecidas por la autoridad.

Una correcta regulación de la intensidad es esencial para gatillar efectos positivos inducidos por el ejerci- cio físico (EF). Los métodos clásicos son basados en porcentajes del consumo de oxígeno máximo (o peak), la frecuencia cardíaca (FC) máxima o de reserva. No obstante, esos métodos requieren condiciones técnicas que dificultan su aplicación en el hogar. En este contexto la percepción del esfuerzo (PE) surge como una alternativa sencilla y adecuada para regular con exactitud la intensidad del EF, sin la necesidad de recursos técnicos.

La PE corresponde a la percepción subjetiva del esfuerzo proveniente de los cambios fisiológicos que sufren los sistemas: cardiovascular, muscular y metabólico durante el ejercicio. De manera interesante, una de las principales ventajas de esta variable surge de la posibilidad de autorregular el esfuerzo mediante métodos que permiten la traducción física de un fenómeno perceptivo, como es la PE (por ejemplo: mediante el empleo de una escala de categorías), lo cual ha motivado la construcción de diversos instrumentos de valoración que han sido adaptados y validados en población infantil ${ }^{3}$.

En nuestro país la escala EPInfant fue desarrollada y validada en niños, a partir de los 8 años de edad, mostrando adecuadas propiedades de medición que han permitido su recomendación y utilización en el contexto clínico, educacional y recreacional ${ }^{3}$. En esta línea, nuestro grupo ha publicado recientemente dos artículos en los que se demuestra la efectividad de las estrategias perceptuales para autorregular la intensidad del ejercicio en niños, entre 8 y 12 años. Es así como Rodríguez-Núñez y cols. ${ }^{4}$, mediante un ensayo de regulación perceptual, demostraron que las categorías perceptivas 6 y 9 de la escala EPInfant reproducen intensidades que alcanzan una FC de 163,5 y 176,2 lat/ min, respectivamente, con niveles moderados a altos de confiabilidad. Más tarde Hernández y cols. ${ }^{5}$, mediante una prueba denominada "Talk Test", demostraron por primera vez en niños entre 8 y 12 años, que la capacidad para verbalizar palabras en voz alta por 30 segundos durante el ejercicio (ej. 31 primeras palabras del himno nacional de Chile), se relaciona estrecha-

Correspondencia:

Iván Rodríguez-Núñez

ivanrodriguez@udec.cl 


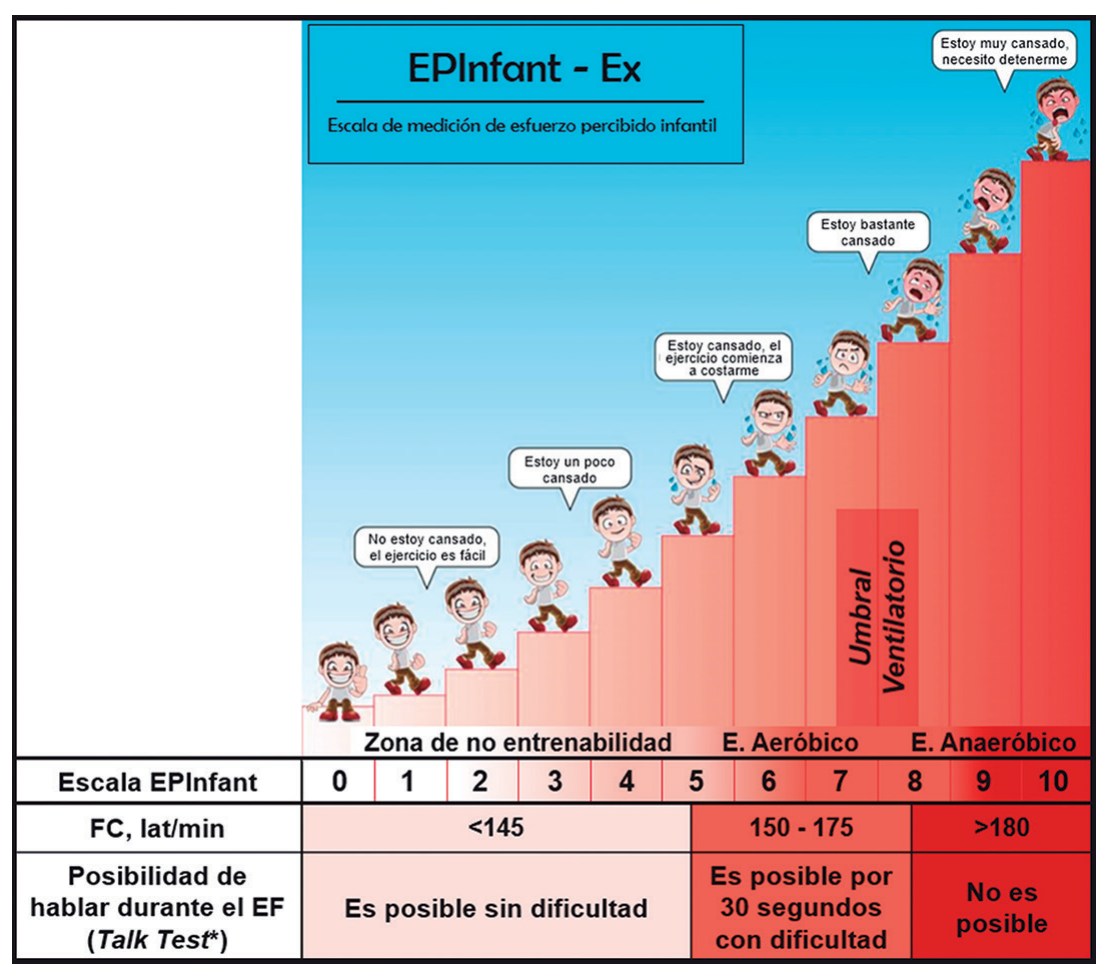

Figura 1. Escala EPInfant modificada (EPInfant-Ex). El instrumento muestra las zonas de entrenamiento y la respuesta fisiológica, según puntaje de la escala EPInfant. (Reproducción y modificación autorizada por el autor. Ref. 3). FC: Frecuencia cardiaca. EF: Ejercicio físico; *El Talk Test permite determinar la intensidad del EF equivalente al umbral ventilatorio, mediante la percepción de dificultad respiratoria al verbalizar en voz alta un set de palabras pre definidas, por 30 segundos. La intensidad de EF a la cual aparece este fenómeno sería equivalente al umbral ventilatorio (Ref. 5). mente con la FC y la PE. Específicamente se observó que a un nivel de intensidad equivalente a 7 puntos de la escala EPInfant y/o 176,8 lat/min (87,9\% de la FC máx.), los niños percibieron una sensación de disconfort respiratorio (durante la verbalización) que pudo ser reproducible en otra situación de ejercicio, a la misma intensidad experimentada previamente ${ }^{5}$.

Estas observaciones muestran que los niños, al aprender estas sencillas técnicas de autorregulación perceptual, podrían reproducir la intensidad del EF con una precisión aceptable y en niveles apropiados para mantener una salud optima, sin la necesidad de emplear algún equipamiento adicional.

Por consiguiente, y de acuerdo a la evidencia citada, sería posible recomendar la ejecución de EF autorregulado a una intensidad igual o mayor a 6 puntos de la escala EPInfant, y/o a una intensidad tal que exista dificultad para verbalizar (recitar o cantar) palabras durante 30 segundos continuados. En la figura 1 se muestra la escala EPInfant modificada (EPInfant-Ex), con las zonas de entrenamiento según puntaje de $\mathrm{PE}$ y el rango de FC reproducida perceptualmente. Estos métodos sencillos de prescripción pueden ser aplicados a las diferentes modalidades de ejercicio domiciliario con efecto demostrado en la evidencia, como: saltar la cuerda, cama elástica, ejercicios de escalón y danza.

Se espera que estos antecedentes sean de ayuda para fundamentar la indicación de actividad física libre, que alcance una intensidad umbral (autorregulada perceptualmente) y en una modalidad tal que produzca sentimientos de gratificación en los niños, como son las actividades de juego libre, lúdico y espontaneo. Ello contribuirá a una mejor adherencia y mantención de este tipo de actividades durante los periodos de cuarentena por COVID-19, pudiéndose prolongar incluso después de las medidas de confinamiento.

Iván Rodríguez-Núñez

${ }^{a}$ Departamento de Kinesiología, Facultad de Medicina, Universidad de Concepción, Chile.

\section{Referencias}

1. Johns Hopkins University. Coronavirus COVID-19 Global Cases by the Center for System Science and Engineering (CSSE) 2020. Available from: https://coronavirus.jhu.edu/ map.html.

2. Booth FW, Roberts CK, Thyfault JP, Ruegsegger GN, Toedebusch RG. Role of Inactivity in Chronic Diseases: Evolutionary Insight and Pathophysiological Mechanisms. Physiol Rev. 2017;97(4):1351-402.

3. Rodríguez I, Gatica D. Percepción de esfuerzo durante el ejercicio: ¿Es válida su medición en la población infantil? Rev Chil Enferm Respir. 2016;32:25-33.

4. Rodríguez-Núñez I, Luarte-Martínez S, Landeros I, Ocares G, Urízar M, Henríquez MJ, et al. Evaluación de la escala EPInfant para la auto-regulación perceptual de la intensidad del ejercicio en niños sanos. Rev Chil Pediatr. 2019;90:422-8.

5. Hernández D, Pacheco N, Poblete I, Tórres H, RodríguezNúñez I. Evaluación del Talk Test como método para estimar la intensidad del ejercicio en niños sanos. Retos. 2019; (37): 303-8. 